\title{
Chemical Gas Sensor Based on a Flexible Capacitive Microwave Transducer Associated with a Sensitive Carbon Composite Polymer Film ${ }^{+}$
}

\author{
Prince Bahoumina 1,*, Hamida Hallil ${ }^{1}$, Jean-Luc Lachaud ${ }^{1}$, Aymen Abdelghani ${ }^{2}$, Kamel Frigui ${ }^{2}$, \\ Stephane Bila ${ }^{2}$, Dominique Baillargeat ${ }^{2}$, Qing Zhang ${ }^{3}$, Phillipe Coquet ${ }^{3,4}$, Carlos Paragua ${ }^{4}$, \\ Emmanuelle Pichonat ${ }^{4}$, Henri Happy ${ }^{4}$, Dominique Rebière ${ }^{1}$ and Corinne Dejous ${ }^{1}$ \\ 1 University of Bordeaux, Bordeaux INP, CNRS, IMS UMR 5218, F-33405 Talence, France; \\ hamida.hallil-abbas@u-bordeaux.fr (H.H.); jean-luc.lachaud@ims-bordeaux.fr (J.-L.L.); \\ dominique.rebiere@ims-bordeaux.fr (D.R.); corinne.dejous@ims-bordeaux.fr (C.D.) \\ 2 University of Limoges/CNRS, XLIM UMR 7252, F-87060 Limoges, France; \\ aymen.abdelghani@unilim.fr (A.A.); kamel.frigui@xlim.fr (K.F.); stephane.bila@xlim.fr (S.B.); \\ dominique.baillargeat@xlim.fr (D.B.) \\ 3 CINTRA, CNRS/NTU/THALES, UMI 3288, Singapore 637553, Singapore; EQZHANG@ntu.edu.sg (Q.Z.); \\ philippe.coquet@iemn.univ-lille1.fr (P.C.) \\ 4 University of Lille, CNRS, IEMN UMR 8520, F-59652 Villeneuve d'Ascq, France; \\ carlos.paragua@iemn.univ-lille1.fr (C.P.); emmanuelle.pichonat@iemn.univ-lille1.fr (E.P.); \\ henri.happy@iemn.univ-lille1.fr (H.H.) \\ * Correspondence: prince.bahoumina@ims-bordeaux.fr; Tel.: +33-054-0002-881 \\ + Presented at the Eurosensors 2017 Conference, Paris, France, 3-6 September 2017.
}

Published: 18 August 2017

\begin{abstract}
This communication presents results on the feasibility of an inkjet printed chemical gas sensor suitable for a real time multi-sensing platform. The prototype targets volatile organic compounds (VOCs) such as ethanol vapor to monitor environmental pollution. The designed and ink-jet printed microwave sensor is presented. Preliminary results have shown the influence of ethanol vapor on the electrical properties of the sensor at microwave frequency range. The sensor's sensitivity to ethanol vapor has been estimated to $-2.48 \mathrm{kHz} / \mathrm{ppm}$. The final aim of this work is to develop a low cost sensor for Internet of Things (IoT) applications.
\end{abstract}

Keywords: chemical gas sensor; electromagnetic transduction; inkjet printing; flexible substrate; carbon materials; microwave device; resonator

\section{Introduction}

Atmospheric environmental pollution caused 7 million deaths in the year 2012 as stated by the World Health Organization (WHO) [1] (pp. 3-4). This is related, among other things, to the complexity and diversification of pollutants, known as a source of irritation and decreased lung capacity. Some of these pollutants are even considered carcinogenic, among them VOCs, which is why they were chosen as target compounds. In this context, we propose a low cost chemical sensor for real-time monitoring and quantification, combining an electromagnetic transduction resonator with a sensitive layer, in the frequency range up to $6 \mathrm{GHz}$. In addition, the device will be integrated in an IoT and embedded system solution in the future, offering interesting prospects for the proliferation of detection and control communicating wireless networks sites. The device is fabricated on a flexible paper substrate by inkjet printing technology [2] (pp. 876-886), [3-5] (pp. 425-435). We present the theoretical study, the results of experimental characterizations, before concluding and opening on perspectives of the sensor based on two bandpass resonators. 


\section{Theoretical Study}

The studied platform is based on two capacitive resonators in order to provide differential detection. One resonator is functionalized with a sensitive material and constitutes the sensitive channel, while the other is considered as a reference channel and compensates the variations not related to the sensitive material. Each channel consists of two parallel networks of 50 electrodes each, spaced with a gap (L2) of $300 \mu \mathrm{m}$. The geometry and configuration of this prototype is shown in Figure 1

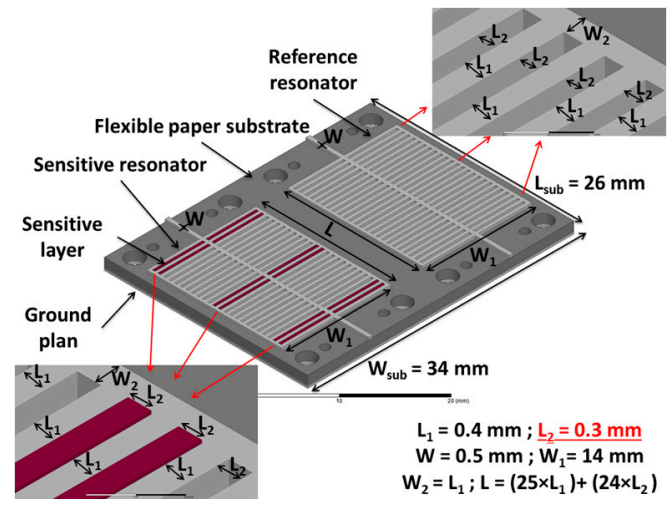

(a)

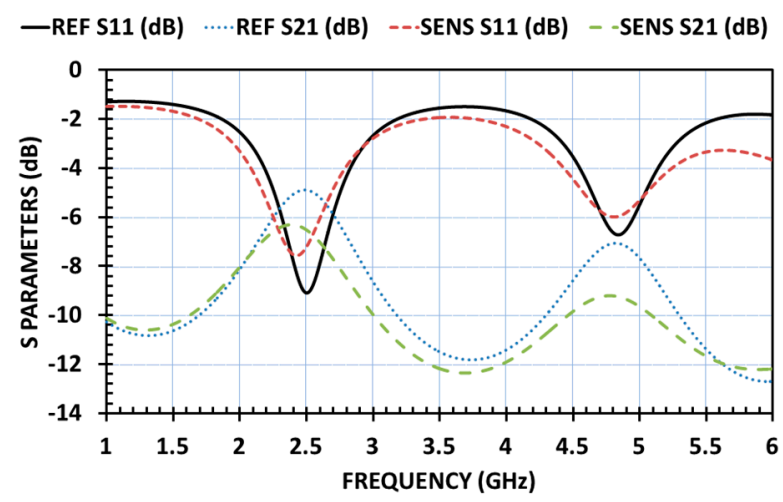

(b)

Figure 1. (a) Overview of the device geometry and (b) Simulated S parameters.

The working principle is based on the perturbation of the electromagnetic field due to the presence of vapor. The target species are absorbed on the sensitive layer and modify the electrical properties of the materials thus the electrical behavior of the device [2,3] (pp. 876-886). Using ANSYSHFSS software which delivers 3-D full-wave simulation for RF components, we perform the simulation with paper as substrate (permittivity 3.05 and dielectric loss 0.089 at $4.3 \mathrm{GHz}$ ), silver nanoparticles as metallized lines, conductive polymer poly (3,4-ethylenedioxythiophene) polystyrene sulfonate with multi walled carbon nanotubes (PEDOT: PSS-MWCNTs) as sensitive layer. The material were considered as homogeneous in a first approximation, their parameters were measured and presented previously, in $[3,6,7]$. Figure $1 \mathrm{~b}$ shows the $S$ parameters of the simulated device. Each resonator has two resonant modes in the frequency range up to $6 \mathrm{GHz}$, around 2.49 and $4.83 \mathrm{GHz}$, respectively. The simulation of the addition of 5 layers of the sensitive material induces a frequency shift close to $-100 \mathrm{MHz}$ for each mode.

The distribution of the electric field $\mathrm{E}$ for both resonant modes of the reference channel is shown in Figure 2. To ensure good sensitivity, the 5-layers sensitive film was placed on the three zones where the E field is more intense, enhancing a capacitive disturbance effect for the first and second modes.

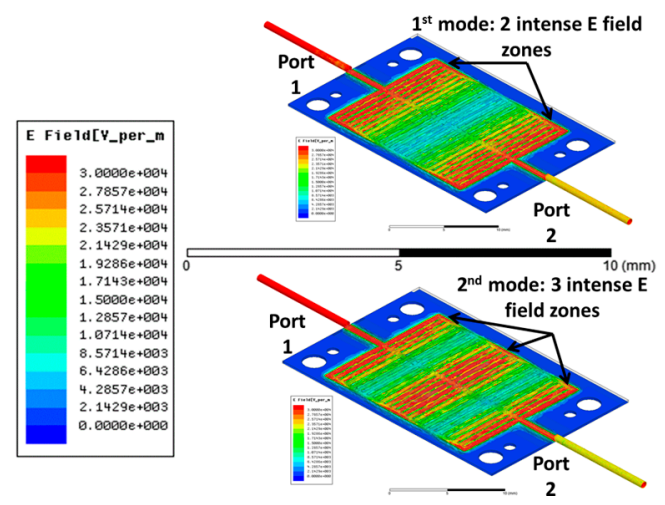

Figure 2. Distribution of the electric field E for both resonant modes. 


\section{Experimental Study and Discussion}

The sensor was fabricated on flexible paper substrate using inkjet printing technology (Dimatix inkjet printer 2800 series). We used an Epson photo paper with a thickness equal to $260 \mu \mathrm{m}$ for the substrate, a silver nanoparticles solution ink (JSB $25 \mathrm{HV}$ ) for the metallized geometries and a composite polymer solution doped with 1 to $1.2 \%$ of multi-wall carbon nanotubes ink (Poly-Ink HC) for the sensitive film. Figure 3a shows the manufactured device with 5 layers of the sensitive material as indicated in part 2, and Figure $3 \mathrm{~b}$ shows the experimental $\mathrm{S}$ parameters measured over the frequency range from 1 to $6 \mathrm{GHz}$ with the vector network analyzer (VNA) MS2026B, calibrated with 4001 points. We will focus on the first mode only, within the reduced frequency range $(2-4 \mathrm{GHz})$. The behavior of this first mode for both resonators under ethanol vapor is presented in the next part. Further studies are planned in order to remove the ripples disturbing the second mode.

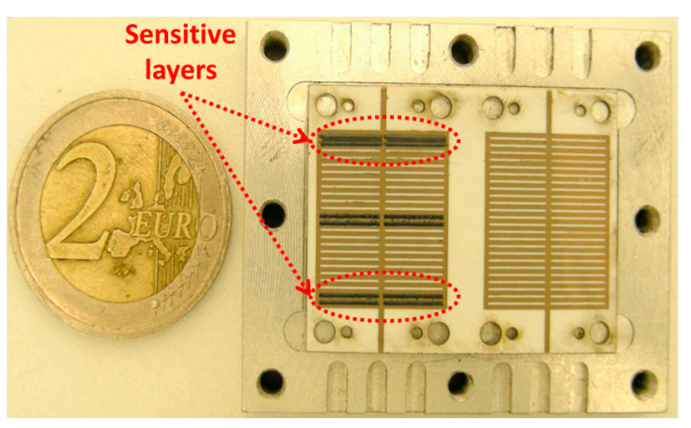

(a)

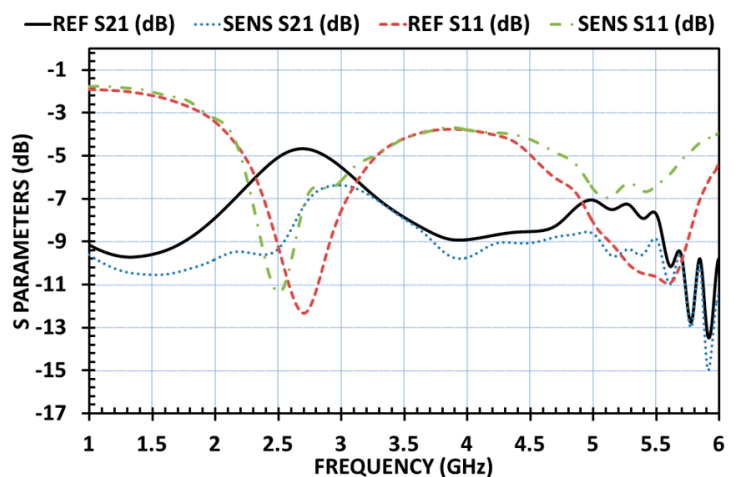

(b)

Figure 3. (a) Experimental device on paper mounted on the test cell basement; (b) Experimental $S$ parameters.

The experimental detection configuration is mainly based on a vapor generator (CALIBRAGE PUL 110), which is used to generate and control the concentrations of the target vapor from a liquid contained in a heated bottle. The vapor particles are transported by nitrogen as a carrier gas at a constant flow rate of $0.112 \mathrm{~L} / \mathrm{min}[3,8]$. A sequence of target vapor concentrations (C) was delivered on the two resonators of a device placed in the low cost test cell. Ethanol was used as model target vapor, using the sequence: $0,500,0,500,0,1000,0,1000,0,2000 \mathrm{ppm}$ for $10 \mathrm{~min}$ each concentration step, carried out after an initial rinsing step under nitrogen for $450 \mathrm{~min}$ to ensure stability at room temperature. The $0 \mathrm{ppm}$ concentration corresponds to nitrogen exposure only. The relative permittivity of the ethanol is about 24.5 at $25^{\circ} \mathrm{C}$, compared to the permittivity of nitrogen which is close to 1 , that may cause a change in the environment of the sensor and will thus modify the electrical properties of the sensitive layer, resulting in an additional variation of the sensing channel compared to the reference one [9]. All detections are carried out under the same conditions, at a fixed temperature equal to $26^{\circ} \mathrm{C}$ and a humidity of $32 \% \mathrm{RH}$.

At this point we recall that we focus on the behavior of the first resonance mode of the device. The effects of the interaction of the ethanol vapor with the electromagnetic fields of the resonators are thus observed in the reduced frequency range between 2 and $4 \mathrm{GHz}$ that is recorded with the same VNA calibrated with 4001 points. Figure 4a illustrates the effect of the ethanol vapor on the resonant frequencies. These measurements correspond to the real time dynamic detection of different ethanol vapor concentrations (from 0 to $2000 \mathrm{ppm}$ ). While the frequency related to the minimum insertion losses of the reference resonator (FrS21r) increases slightly with the ethanol concentration, the frequency of the sensitive resonator (FrS21s) decreases significantly at the same time. The steady state frequencies at the end of each 10 minutes step as function of the concentration are reported on Figure $4 \mathrm{~b}$. On both figures are superimposed the resulting differential signal, by subtracting the response of the reference from that of the sensitive line: $\Delta \mathrm{F} \_\mathrm{S} 21=\mathrm{FrS21s}-\mathrm{FrS21}$. This leads to the estimation of a high level sensitivity $(-2.48 \mathrm{kHz} / \mathrm{ppm})$ which is promising for future applications of the sensor. 


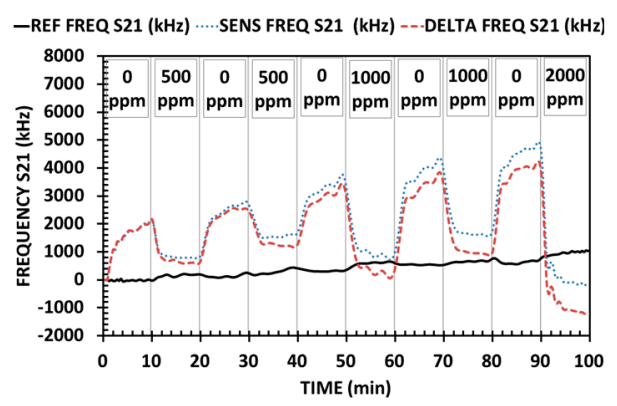

(a)

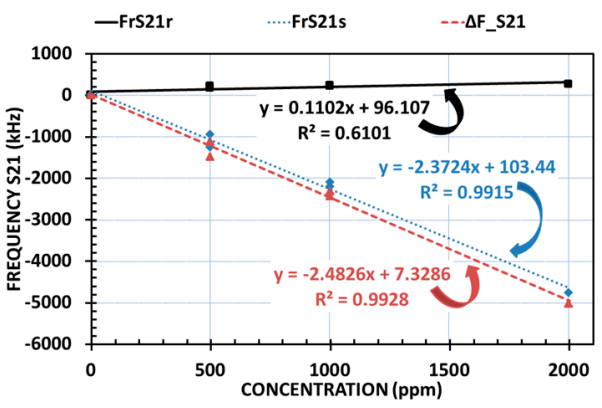

(b)

Figure 4. (a) Real-time frequency variation with different ethanol vapor concentrations; (b) Resulting experimental steady state frequency shift (monitored at $10 \mathrm{~min}$ ) and sensitivity.

\section{Conclusions}

The feasibility of a fully inkjet printed chemical gas sensor based on a flexible capacitive microwave transducer associated with a sensitive carbon composite polymer film has been demonstrated by the theoretical study based on finite element tool for electromagnetic simulations and by relevant experimental study. The sensitivity might be further improved by increasing the thickness of the sensitive material, or by its further functionalization. Future studies will aim to improve the understanding of the physical phenomena that are involved. However, the high effective surface area of the proposed device could be compatible with other sensitive materials, within a large range of electrical conductivity. It is thus very interesting for the development of various chemical gas sensors based on an integrated microwave device.

Acknowledgments: We would like to thank the National Research Agency for its support (CAMUS project, ANR-13-BS03-0010) and the French Embassy of Singapore (Merlion project), as well as the RENATECH national network for its technological support.

Conflicts of Interest: The authors declare no conflict of interest.

\section{References}

1. Open-Ended CPR. Healthy Environment, Healthy People; United Nations Environment Programme: Nairobi, Kenya, 2016.

2. Rydosz, A.; Maciak, E., Wincza, K., Gruszczynski, S. Microwave-based sensors with phthalocyanine films for acetone, ethanol and methanol detection. Sens. Actuators B Chem. 2016, 237, 876-886.

3. Bahoumina, P.; Hallil, H.; Lachaud, J.L.; Abdelghani, A.; Frigui, K.; Bila, S.; Baillargeat, D.; Ravichandran, A.; Coquet, P.; Paragua, C.; et al. Microwave flexible gas sensor based on polymer multi wall carbon nanotubes sensitive layer. Sens. Actuators B Chem. 2017, 249, 708-714.

4. Tentzeris, M.M.; Kim, S.; Traille, A.; Aubert, H.; Yoshihiro, K.; Georgiadis, A.; Collado, A. Inkjet-printed RFID-enabled sensors on paper for IoT and "Smart Skin" applications. In Proceedings of the 21st International Conference on Applied Electromagnetics and Communications (ICECom), Dubrovnik, Croatia, 14-16 October 2013.

5. Aubert, H.; Chebila, F.; Jatlaoui, M.; Thai, T.; Hallil, H.; Traille, A.; Bouaziz, S.; Rifaï, A.; Pons, P.; Menini, P.; et al. Wireless sensing and identification based on radar cross section variability measurement of passive electromagnetic sensors. Ann. Telecommun. 2013, 68, 425-435.

6. Paragua, C.; Frigui, K.; Bila, S.; Baillargeat, D. Study and characterization of CNT Inkjet printed patterns for paper-based RF components. In Proceedings of the 2015 European Microwave Conference (EuMC), Paris, France, 7-10 September 2015; pp. 861-864.

7. Pasha, A.; Roy, A.S.; Murugendrappa, M.V.; Al-Hartomy, O.A.; Khasim, S. Conductivity and dielectric properties of PEDOT-PSS doped DMSO nano composite thin films. J. Mater. Sci. Mater. Electron. 2016, 27, 8332. 
8. Nikolaou, I.; Hallil, H.; Deligeorgis, G.; Conedera, V.; Garcia, H.; Dejous, C.; Rebière, D. Novel SAW gas sensor based on graphene. In Proceedings of the 2015 30th Symposium on Microelectronics Technology and Devices (SBMicro), Salvador, Brazil, 31 August-4 September 2015.

9. Abdolrazzaghi, M.; Zarifi, M.H.; Daneshmand, M. Sensitivity enhancement of split ring resonator based liquid sensors. In Proceedings of the 2016 IEEE Sensors, Orlando, FL, USA, 30 October-3 November 2016.

(C) 2017 by the authors. Licensee MDPI, Basel, Switzerland. This article is an open access article distributed under the terms and conditions of the Creative Commons Attribution (CC BY) license (http://creativecommons.org/licenses/by/4.0/). 